\title{
Self-assembly of magnetic balls: From chains to tubes
}

\author{
René Messina* and Lara Abou Khalil \\ Laboratoire de Chimie et Physique - Approche Multi-Echelle des Milieux Complexes (LCP-A2MC), \\ Institut de Chimie, Physique et Matériaux (ICPM), Université de Lorraine, 1 Boulevard Arago, 57070 Metz, France \\ Igor Stanković \\ Scientific Computing Laboratory, Institute of Physics Belgrade, University of Belgrade, 11080 Belgrade, Serbia
}

(Received 22 July 2013; published 9 January 2014)

\begin{abstract}
The self-assembly of spherical magnets (magnetic balls) is addressed theoretically. Minimal energy structures are obtained by optimization procedures as well as Monte Carlo computer simulations. Three typical shapes are obtained depending on the number of constitutive magnets $N$. In the regime of small $N$, chains are stable as dimers or trimers (i.e., $N \leqslant 3)$, then rings become stable for $(4 \leqslant N \leqslant 13)$ where dipole vectors adopt a vortexlike arrangement. A major finding concerns the stacking of rings as soon as $N$ is large enough $(N \geqslant 14)$. The number of stacked rings is found to increase as $N^{2 / 3}$, leading to a tubular structure at large $N$. All the relevant predicted shapes are experimentally reproduced by manipulating millimetric magnets.
\end{abstract}

DOI: 10.1103/PhysRevE.89.011202

PACS number(s): 41.20.Gz, 64.75.Yz, 05.65.+b

Human beings have always been fascinated with manipulating and assembling magnets [1]. Many valuable applications of such systems can be found in different areas. For instance, in nanotechnology, self-assembled mixtures of magnetic nanoparticles can lead to very strong magnets [2,3]. The interaction between magnetically patterned planar sheets can yield remarkable three-dimensional (3D) objects with high potential for the microfabrication of 3D electronic devices [4]. In biology, some bacteria own a permanent magnet and they are called magnetotactic bacteria [5]. Under an external magnetic field, the latter form chainlike structures [6]. From a more physical perspective, elastic interactions of cells can be mapped onto effective dipolarlike interactions [7].

From a theoretical point of view, understanding magnetic interactions and in particular the interaction between magnets is very challenging due to its long range and anisotropy. In this spirit, the pioneering theoretical work of Jacobs and Beans [8] and later that of de Gennes and Pincus [9] shed some light on the microstructure of self-assembled (spherical) magnets. Clearly, solely the microstructural behavior for a very small number of magnets $(N \leqslant 4)$ is understood at zero temperature. More recently, microstructures of dipolar fluids have been thoroughly studied by computer simulations [10-12], and again a key feature is the formation of chains at finite temperature. With all that being said, to date, the self-assembly (at zero temperature) of magnetic balls has yet to be properly addressed. The goal of the present contribution is to elucidate this fascinating problem in a physically simple and transparent framework.

In this Rapid Communication, we predict the self-assembly of spherical magnets (typically in chains, rings, and tubes or cylinders) possessing a minimal energy for virtually all $N$. Besides, commercial millimeter-sized magnets (commonly called "buckyballs" or "neocubes"-see the inset in Fig. 1) are employed to reproduce and confirm the calculated ground state structures.

The length scale of the system is provided by the diameter $d$ of the magnet (see Fig. 1), and the dipole strength is $m$.

*rene.messina@univ-lorraine.fr
The potential energy of interaction $U\left(\vec{r}_{12}\right)$ between two (ideal) magnetic balls whose centers are located at $\vec{r}_{1}$ and $\vec{r}_{2}$ (see Fig. 1) can be written as

$$
U\left(\vec{r}_{12}\right)=C \frac{1}{r_{12}^{3}}\left[\vec{m}_{1} \cdot \vec{m}_{2}-3 \frac{\left(\vec{m}_{1} \cdot \vec{r}_{12}\right)\left(\vec{m}_{2} \cdot \vec{r}_{12}\right)}{r_{12}^{2}}\right]
$$

for $r_{12} \geqslant d$ or $\infty$ otherwise, where $C$ represents a constant that depends on the intervening medium, and $r_{12}=\left|\vec{r}_{12}\right|=$ $\left|\vec{r}_{2}-\vec{r}_{1}\right|$. It is convenient to introduce the energy scale defined by $U_{\uparrow \uparrow} \equiv \frac{C m^{2}}{d^{3}}$ that physically represents the repulsive potential value for two parallel dipoles at contact, standing side by side, as clearly suggested by the notation. Thereby the reduced potential energy of interaction $u_{N}$ (per magnet) of $N$ magnetic balls reads

$$
u_{N}=\frac{1}{N} \sum_{i=1}^{N-1} \sum_{j=i+1}^{N} \frac{U\left(\vec{r}_{i j}\right)}{U_{\uparrow \uparrow}} \quad\left(r_{i j} \geqslant d\right) .
$$

It is precisely this function (2) that has to be minimized by carefully respecting the nonoverlapping conditions. Note that upon searching the minimal energy, five variables per magnet are involved (see also Fig. 1): three Cartesian coordinates $(x, y, z)$ for the center, and two angular parameters $(\theta, \phi)$ for the unit vector defining the direction of the dipole. Two fully different numerical routes were employed to calculate the energy minimum of the system: (i) standard minimization routines (e.g., penalty method [13]) and (ii) Monte Carlo (MC) simulations [14]. In the latter case, a gentle quench from finite to zero temperature is applied so as to avoid an early trapping in local minima. When zero temperature is reached, only trial moves leading to lower energies are accepted as long as possible. In order to increase the chance of finding the ground state, typically 1000-10 000 starting configurations were considered. The winning structure is then that possessing the lowest final energy. By doing so in both methods (standard minimization and MC techniques), a quantitative agreement is achieved, giving us confidence in our results.

An overview of our results is provided in Fig. 2, where typical stable structures as a function of $N$ are depicted. 


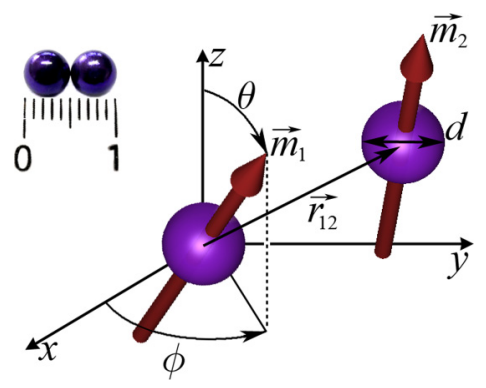

FIG. 1. (Color online) Scheme of two interacting spherical magnets. For convenience, one magnet is placed at the origin so that $\vec{r}_{1}=0$. The polar and azimuthal angles $(\theta, \phi)$ are explicitly shown for $\vec{m}_{1}$. The inset (top left corner) of a 1 ruler centimeter exemplifies the millimeter-sized magnets utilized in our experiments.

Upon increasing $N$, one-dimensional structures are found (chains with $N=2,3$ ), then two-dimensional ones (single rings with $4 \leqslant N \leqslant 13$ ), and finally three-dimensional ones (multiple rings with $N \geqslant 14$ ) (see Fig. 2). Interestingly and strikingly, as shown in Fig. 2, all the predicted minimal energy structures are reproduced experimentally with magnetic balls. More specifically, at $N=2,3$ the well-known arrangement of a single file of the dipoles is found (see Fig. 2). As also shown in Fig. 2, for $4 \leqslant N \leqslant 13$, regular polygons with touching consecutive spheres are obtained and are also referred to as rings. The dipole vectors are tangential to the inscribed circular loop (see, e.g., $N=13$ in Fig. 2), reminiscent of a vortex. As we show in Fig. 2, for $N=14$, a remarkable transition from a single-ring to a double-ring structure occurs. The projected structure along the axis symmetry corresponds to a regular tetradecagon (14 sides). Thereby each constitutive ring is identical to the single-ring ground state at $N=7$. By analogy, with crystalline solids this double heptagon ordering can be seen as an $A B$ stacking. The first irregular structure appears when $N=15$ due to some incommensurability between the two constitutive rings that are now made up of eight and seven magnets. Typically, in this scenario where a perfect $A B$ stacking is prohibited, the dipole vectors are still curling around a nearly planar circular loop, except a dimer of magnets that is (slightly) vertically shifted (see Fig. 2).

We now would like to provide a more quantitative analysis by addressing the energy behavior for different shapes, namely, chains, rings, and stacks of rings. Certainly the most simple and intuitive situation concerns a straight chain of dipoles in a row. Thereby, for $N$ aligned magnets (i.e., $\rightarrow \rightarrow \cdots \rightarrow$ ), the reduced energy per magnet $u_{N}^{\text {(chain) }}$ is simply given by

$$
u_{N}^{\text {(chain) }}=-\frac{2}{N} \sum_{i=1}^{N-1} \sum_{j=i+1}^{N} \frac{1}{(j-i)^{3}} .
$$

For an infinite chain $(N=\infty)$, the reduced energy per magnet $u_{\infty}^{\text {(chain) }}$ is then given by

$$
u_{\infty}^{\text {(chain) }}=-2 \sum_{k=1}^{\infty} \frac{1}{k^{3}}=-2 \zeta(3) \simeq-2.404,
$$

with $\zeta(n)=\sum_{k=1}^{\infty} \frac{1}{k^{n}}$ standing for the Riemann zeta function. Since the single-ring structure is the crucial finding in the regime of small $N$, it is handy to have an analytical expression
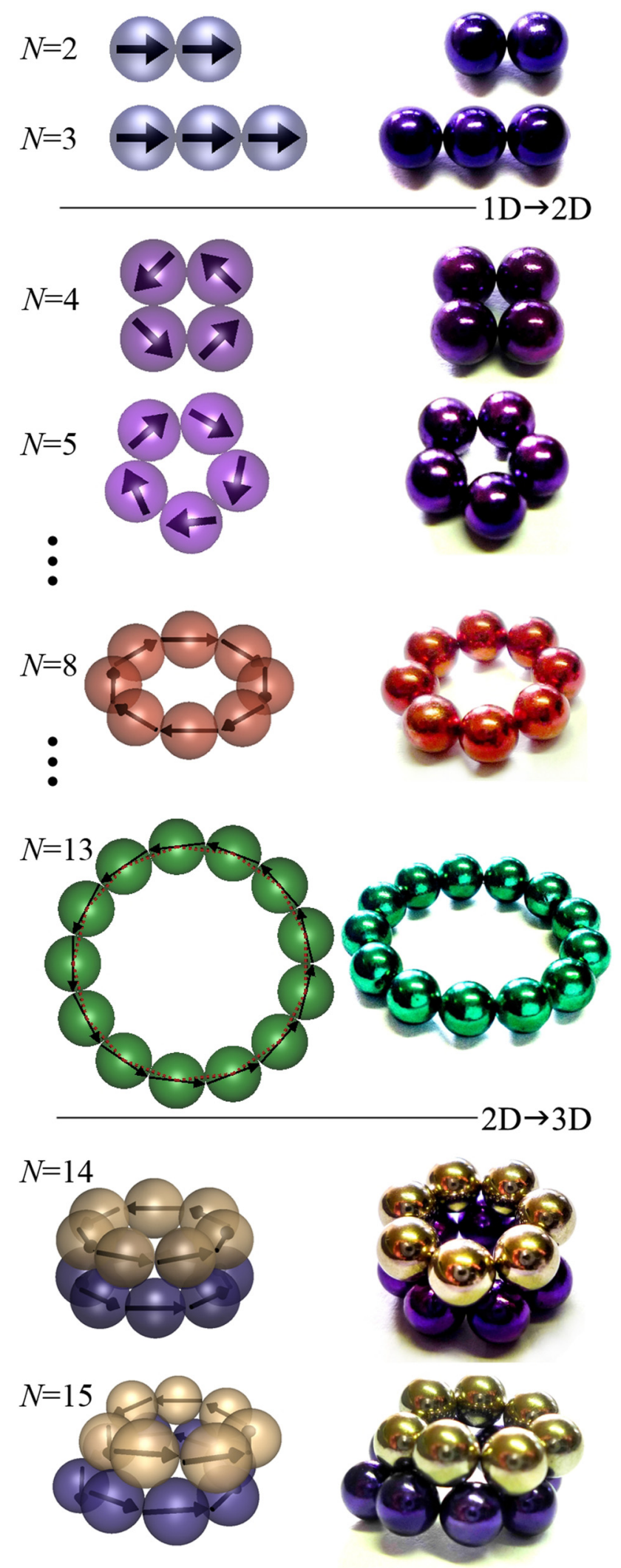

FIG. 2. (Color online) Minimal energy configurations for $2 \leqslant$ $N \leqslant 15$. The left column shows configurations (including the dipole vectors) stemming from the minimization calculations. The right column shows stable experimental configurations obtained by manipulating millimetric magnets. The spatial dimension $(1 \mathrm{D}, 2 \mathrm{D}$, 3D) transitions are also indicated. 


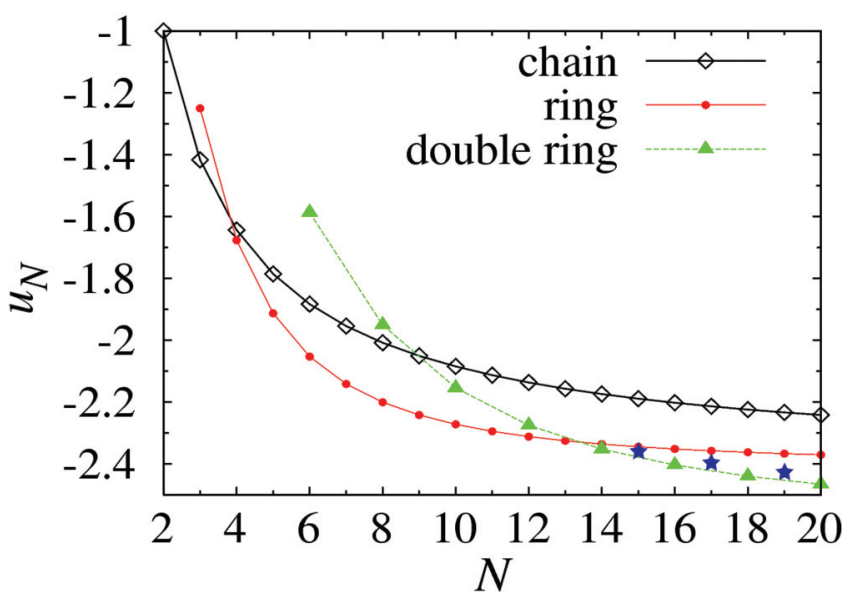

FIG. 3. (Color online) Reduced energy profiles $u_{N}$ as a function of the number of magnets $N$ for different shapes: chain $(\diamond)$, ring $(\bullet)$, and double ring $(\boldsymbol{\Lambda})$. The lines only serve as guides to the eye. Stable irregular double-ring structures obtained at $N=15,17,19$ are denoted by $\star$.

for the corresponding energy $u_{N}^{\text {(ring) }}$. Taking into account the symmetry of the annular states, one arrives at the (exact) following expression:

$$
u_{N}^{\text {(ring) }}=-\frac{1}{4} \sin ^{3}\left(\frac{\pi}{N}\right) \sum_{k=1}^{N-1} \frac{3+\cos \left(\frac{2 \pi k}{N}\right)}{\sin ^{3}\left(\frac{\pi k}{N}\right)} .
$$

Note that $u_{\infty}^{\text {(ring) }}=u_{\infty}^{\text {(chain) }}$, reflecting the fact that at vanishing curvature one recovers the energy per magnet corresponding to an infinite chain. A similar expression as (5) is provided in Ref. [15] for a double ring with $A B$ stacking.

Energy profiles for chains, rings, and double rings are depicted in Fig. 3. The energy profile for the chain structure stems from Eq. (3), whereas that for the ring shape was generated using Eq. (5). The energy profile for double-ring structures was obtained by considering an even number of constitutive magnets allowing a perfect $A B$ stacking. As already mentioned in the the ground state structure analysis (see Fig. 2), for $N \leqslant 14$ all the stable states (i.e., possessing the smallest energy among all possible states at prescribed $N$ ) correspond to ideal structures (i.e., chain, ring, double $A B$ ring) as obtained by independent minimization procedures (e.g., penalty method and MC technique). At the chain-ring transition occurring at $N=4$, in agreement with the earlier work of Jacobs and Bean [8], we have $u_{4}^{\text {(chain) }}=-\frac{710}{432} \simeq$ -1.644 and $u_{4}^{\text {(ring) }} \simeq-1.677$ (see Fig. 3). Then, as shown in Fig. 3, at larger $N$, the single- to double-ring transition takes place for $N=14$ with $u_{14}^{\text {(ring) }} \simeq-2.336$ whereas $u_{14}^{\text {(2-ring) }} \simeq$ -2.362. Interestingly, with $N=14$ one already reaches the asymptotic value $u_{\infty}^{\text {(chain) }} \simeq-2.404$ within $3 \%$. The energy values for the first irregular structures $(N=15,17,19)$ lie between the single- and double-ring energy curves (see Fig. 3), reflecting the fact that defects promote energy penalties. Although not shown here, we were able to locate the double $A B$ ring to triple $A B A$ ring transition at $N=27$ by means of MC simulations [16].

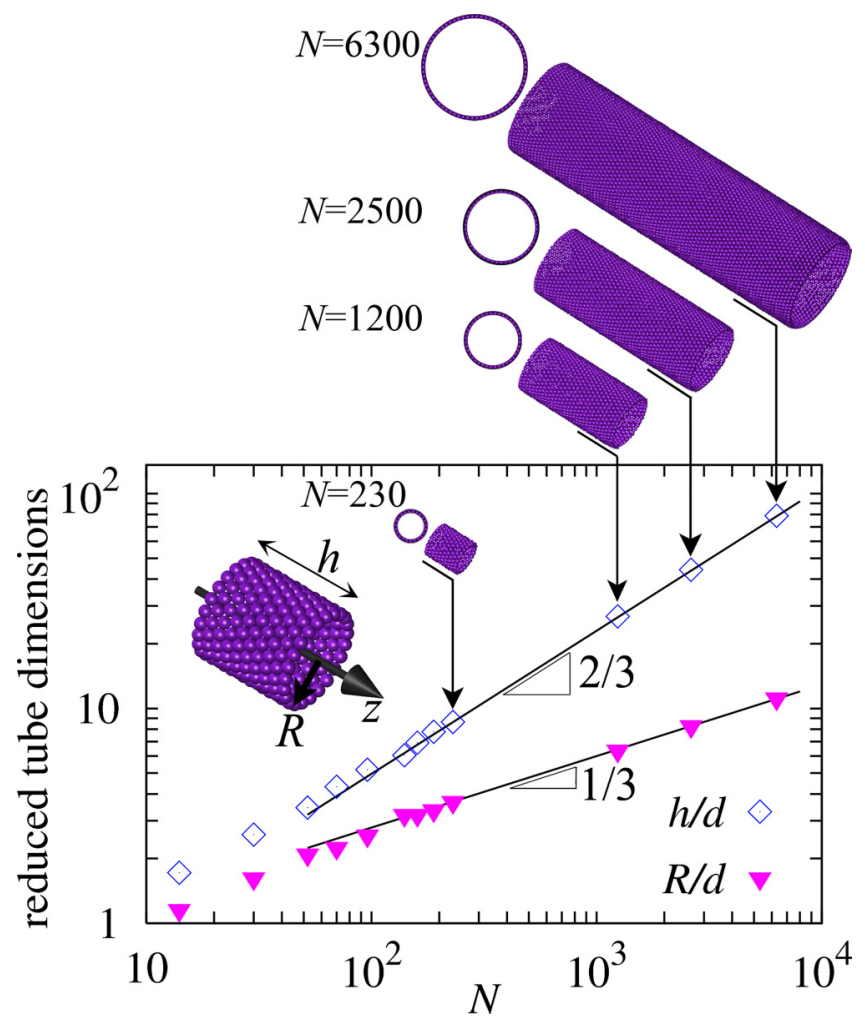

FIG. 4. (Color online) Radius of gyration $R$ and height $h$ of the tubular structure as a function of $N$. The lines correspond to best fits. Microstructures (top and side view) are shown for $N=230$, 1200,2500 , and 6300 to scale. A magnification of the microstructure at $N=230$ is also drawn to illustrate the geometrical tube features ( $h, R, z$ axis).

Having now a good understanding of the self-assembly behavior and especially of the crucial role of ring stacking at small $N$, it is possible to predict analytically the ground state for a vast variety of tubular configurations at large $N$. Clearly, to compute the energy for large $N$, one only considers ideal defect-free stacked rings. This approach is sufficient to capture the main properties of the geometrical properties of the tube as a function of $N$. More specifically, we consider the height $h$ of the tube (parallel to the $z$ axis-see Fig. 4) and its radius of gyration $R$ in the $x y$ plane defined as

$$
R=\sqrt{\frac{1}{N} \sum_{k=1}^{N}\left[\left(x_{k}-x_{\text {c.m. }}\right)^{2}+\left(y_{k}-y_{\text {c.m. }}\right)^{2}\right]}
$$

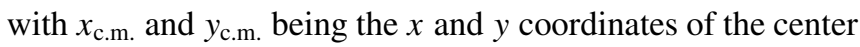
of mass, respectively. The profiles of $R$ and $h$ as a function of $N$ are sketched in Fig. 4. Interestingly, the height $\left(h / d \sim N^{2 / 3}\right)$ grows faster than the radius $\left(R / d \sim N^{1 / 3}\right)$ [17], leading to the following scaling law for large $N$ :

$$
h / R \sim N^{1 / 3} .
$$

Note that another interesting scaling law concerning the energy is reported in Ref. [15]. More specifically, by denoting $u_{N}^{\text {(tube) }}$ the reduced energy per magnet for the tubular structure, it is found that $u_{N}^{\text {(tube) }}-u_{\infty}^{\text {(plane) }} \sim N^{-2 / 3}$. Concerning the crystallization feature at $N \rightarrow \infty$, one has to merely deal 
with an infinite cylinder (in height and radius), meaning that a planar triangular lattice is recovered. In this case the reduced energy per magnet $u_{\infty}^{\text {(plane) }}$ is again given by an exact expression [18], namely, a fast converging double sum,

$$
\begin{aligned}
u_{\infty}^{\text {(plane) }} & =16 \pi^{2} \sum_{k=1}^{\infty} \sum_{l=1}^{\infty} l^{2} \cos (k l \pi) K_{0}(k l \sqrt{3} \pi) \\
& \simeq-2.759
\end{aligned}
$$

with $K_{0}$ being the modified Bessel function of the second kind with zero order. This value, $u_{\infty}^{\text {(plane) }} \simeq-2.759$, has to be compared with that of an infinite ring (or chain), $u_{\infty}^{\text {(chain) }} \simeq$ -2.404 , proving an enhanced stability upon stacking.

In summary, we have demystified the intriguing selfassembly of magnetic balls. The essential finding is the stacking of rings with curling dipole vectors (i.e., vortex arrangement) as minimal energy configurations. These structures correspond either to zero magnetization $M$ [19] for stacking of identical rings, or finite but very weak magnetization (i.e.,
$M / m \ll 1)$ otherwise. In the regime of a small number of magnets, $4 \leqslant N \leqslant 13$, the single ring (regular polygon) is the most stable structure. At higher $N$, stacking of rings leads to stable tubular structures made up of either (i) slightly deformed rings (with defects) or (ii) perfect (defect-free) identical rings. The latter states correspond to some magic numbers for $N$ that are quite abundant. All these predicted structure classes are reproduced experimentally with millimetric magnetic balls, as demonstrated in this Rapid Communication. Note that a system very similar to ours was recently studied [20] where the macroscopic mechanical properties were emphasized [21]. An interesting future study will deal with the effect of an external applied magnetic field where a rich phase behavior is expected [22].

We thank Igor Kulic for drawing our attention to the recent work of Vella et al. [20]. I.S. acknowledges support received from the Serbian Ministry of Education and Science (ON171017 and III45018) and FP7 projects (PRACE-2IP, PRACE-3IP, and EGI-InSPIRE).
[1] W. Gilbert, in De Magnete (Peter Short, London, 1600) (an English version published by Dover in 1991 is also available).

[2] H. Zeng et al., Nature (London) 420, 395 (2002).

[3] D. J. Sellmyer, Nature (London) 420, 374 (2002).

[4] M. Boncheva et al., Proc. Natl. Acad. Sci. USA 102, 3924 (2005).

[5] R. Blakemore, Science 190, 377 (1975).

[6] E. Alphandery et al., ACS Nano 3, 1539 (2009).

[7] U. S. Schwarz and S. A. Safran, Phys. Rev. Lett. 88, 048102 (2002).

[8] I. S. Jacobs and C. P. Bean, Phys. Rev. 100, 1060 (1955).

[9] P. G. de Gennes and P. A. Pincus, Phys. Kondens. Mater. 11, 189 (1970).

[10] J. J. Weis and D. Levesque, Phys. Rev. Lett. 71, 2729 (1993).

[11] C. Holm and J. J. Weis, Curr. Opin. Colloid Interface Sci. 10, 133 (2005).

[12] L. Rovigatti, J. Russo, and F. Sciortino, Phys. Rev. Lett. 107, 237801 (2011).

[13] L. Assoud and R. Messina, Phys. Rev. E 83, 036113 (2011).
[14] M. P. Allen and D. J. Tildesley, Computer Simulations of Liquids (Clarendon, Oxford, UK, 1987).

[15] See Supplemental Material at http://link.aps.org/supplemental/ 10.1103/PhysRevE.89.011202 for an analytical expression for two stacked rings and the tube energy behavior as a function of $N$.

[16] Note that numerical minimizations with $N \gtrsim 20$ (i.e., over 100 variables) become computationally extremely demanding and virtually unfeasible.

[17] Note that $R h / d^{2} \sim N$ at large $N$, reflecting a uniform magnet distribution on the cylinder surface.

[18] A. Grzybowski and A. Brodka, Mol. Phys. 101, 1079 (2003).

[19] For the sake of the discussion, one defines the magnetization $\vec{M}$ as $\vec{M} \equiv 1 / N \sum_{i=1}^{N} \vec{m}_{i}$ and $M \equiv|\vec{M}|$.

[20] D. Vella, E. du Pontavice, C. L. Hall, and A. Goriely, Proc. R. Soc. A 470, 20130609 (2013).

[21] A scenario for minimal energy structures was also briefly discussed by Vella et al. [20]. However, in Ref. [20], they did not properly take into account the finite size of the chain, leading to qualitatively wrong conclusions such as lower energies for chains than for rings.

[22] R. Messina and I. Stankovic (unpublished). 